\title{
Effectiveness of Tuina manipulation in patients with knee osteoarthritis: study protocol for a randomized controlled trial
}

\section{Zhizhen Lv}

Shanghai University of Traditional Chinese Medicine Yueyang Hospital of Integrated Traditional Chinese Medicine and Western Medicine

\section{Qingguang Zhu}

Shanghai University of Traditional Chinese Medicine Yueyang Hospital of Integrated Traditional Chinese Medicine and Western Medicine

\section{Guangxin Guo}

Shanghai University of Traditional Chinese Medicine Yueyang Hospital of Integrated Traditional Chinese Medicine and Western Medicine

\section{Wuquan Sun}

Shanghai University of Traditional Chinese Medicine Yueyang Hospital of Integrated Traditional Chinese Medicine and Western Medicine

\section{Yanbin Cheng}

Shanghai University of Traditional Chinese Medicine Yueyang Hospital of Integrated Traditional Chinese Medicine and Western Medicine

\section{Lingjun Kong}

Shanghai University of Traditional Chinese Medicine Yueyang Hospital of Integrated Traditional Chinese Medicine and Western Medicine

\section{Min Fang ( $\nabla$ fangmin19650510@163.com )}

Institute of Tuina,Shanghai Institute of Traditional Chinese Medicine,Shanghhai 200437,China;Shanghai University of Traditional Chinese Medicine,Shanghai201203囚China

\section{Zhiwei Wu}

Shanghai University of Traditional Chinese Medicine Yueyang Hospital of Integrated Traditional Chinese Medicine and Western Medicine

\section{Shanda Xu}

Shanghai University of Traditional Chinese Medicine Yueyang Hospital of Integrated Traditional Chinese Medicine and Western Medicine

\section{Xin Zhou}

Shanghai University of Traditional Chinese Medicine Yueyang Hospital of Integrated Traditional Chinese Medicine and Western Medicine

\section{Shuaipan Zhang}


Shanghai University of Traditional Chinese Medicine Yueyang Hospital of Integrated Traditional Chinese Medicine and Western Medicine

\section{Chongjie Yao}

Shanghai University of Traditional Chinese Medicine Yueyang Hospital of Integrated Traditional Chinese Medicine and Western Medicine

\section{Study protocol}

Keywords: Knee osteoarthritis, Effectiveness, Randomized controlled trial, Tuina, manipulation treatment

Posted Date: April 2nd, 2020

DOl: https://doi.org/10.21203/rs.3.rs-20550/v1

License: (c) (1) This work is licensed under a Creative Commons Attribution 4.0 International License. Read Full License 


\section{Abstract}

Background: Knee osteoarthritis (KOA) is a common disabling musculoskeletal disorder. Previous studies showed that Tuina manipulation and health care education were effective treatments for patients with KOA. However, there is no evidence to support the use of one intervention rather than another. The purpose of this study is to evaluate the effectiveness of Tuina manipulation and health care education in the management of pain and dysfunction in patients with KOA.

Methods/Design: This study is a single center, two-arm, open-label RCT. A total of 150 eligible KOA patients will be randomly assigned to the Tuina manipulation group or the health education group in a 1:1 ratio. Patients in the Tuina manipulation group receives a 30-minute 2-step treatment plan, including pain point assessment and manual therapy, which are performed 12 times in 4 weeks. The health education group conducts 45 minutes of lectures and discussions each time, three times a week for four weeks. The primary outcome is the Knee Injury and Osteoarthritis Outcome Score (KOOS). Secondary outcomes include the McGill Pain Questionnaire (MPQ) and 36-item Short-Form Health Survey (SF-36). The results of this study will help clarify the value of Tuina manipulation and health education as treatments for KOA and will highlight any differences in treatment outcomes.

Discussion: The design and methodology of the trial is rigorous and allows the collection of valuable data to assess the efficacy of a specific Tuina regimen in the treatment of KOA. Therefore, the trial will provide a solid foundation for the future clinical research of KOA as well as the future of Tuina therapy.

Trial registration: This trial was registered with Chinese Clinical Trial Registry on 24 February 2020(ChiCTR2000030154), http://www.chictr.org.cn/index.aspx

\section{Background}

Knee osteoarthritis (KOA) is a disease characterized by joint pain, joint inflammation, stiffness and dysfunction [1]. KOA is a worldwide disease that affects human health which needs to spend huge sums of money every year to bring a heavy financial burden to society [2]. Specific conditions contributing to

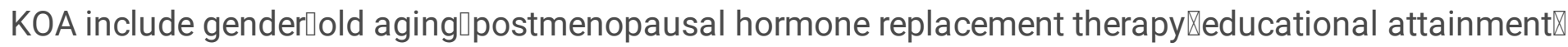
obesity[3].In people over the age of 55 , the prevalence of pain and disability in knee osteoarthritis is $10 \%$, and a quarter of them are severely disabled[4].According to the survey, approximately $14 \%$ of American adults and $40 \%$ of Britons and Australians over the age of 65 suffer from this disease; the prevalence of $\mathrm{KOA}$ is increasing year by year[5].

The pathophysiology of KOA has not been fully established because it is difficult to determine the source of pain. Studies have confirmed that cytokines such as tumor necrosis factor-a and proteases play an important role in the occurrence and development of osteoarthritis. The level of TNF-a in plasma and synovial fluid of patients with osteoarthritis was significantly higher than that of normal people, and the level of TNF-a in synovial fluid was related to the degree of KOA lesions[6-7]. 
The affected joint showed asymmetry narrowing of the joint space, osteosclerosis and/or cystic deformation occurred at the lower end of the cartilage, and extra bone formation at the joint edge are the typical X-ray manifestation of KOA.MRI and CT can be used for early KOA diagnosis, differential diagnosis and clinical research. MRI is characterized by thinning of cartilage thickness, tissue defect, meniscus injury and degeneration, joint effusion, edema and popliteal cyst. CT can observe the stenosis of the affected joint space, subchondral bone sclerosis, cystic changes and osteophyte hyperplasia[8].

In addition, there are several different treatments for controlling this disease, such as intra-articular (IA) corticosteroid (CS) injection, non-steroidal anti-inflammatory drugs (NSAID), opioids, physical therapy (PT), arthroscopy , IA hyaluronic acid (HA) injection\surgery and exercise therapy [9-12]. However, NSAIDs may induce mucosal damage in the upper digestive tract and lower digestive tract[13].Even with the use of gastric protectants, up to three-quarters of patients who use non-steroidal anti-inflammatory drugs suffer from small bowel damage[14]. Opioids also have an addiction risk[15].These treatments can achieve certain effects, but have certain recurrence rates and side effects. And the use of the method depends on the stage of the disease and the specific circumstances of the patient. When basic and medical treatments are not effective, patients can only seek surgery for help.However, surgery requires a lot of economics, which brings huge economic burden to families and society. Therefore, in order to consider the safety and economy of treatment, we need to find a treatment with small side effects and low price.

Tuina is one of physical therapies,which is commonly defined as a long-standing healing art by fingers and strength[16]. When treating KOA with Tuina, the manipulate technique should be gentle and achieve a certain treatment time to relieve pain for optimal clinical efficacy.

As a physical therapy, manipulative therapy has been recommended by many countries as one of the non-drug therapies for the treatment of motor system diseases. Recent clinical studies and systematic reviews have proven that manipulation is safe and effective for the treatment of knee osteoarthritis [1719]. Compared with NSAIDs $₫$ massage therapy can improve functional pain more economically. However, due to differences in treatment accuracy,different treatment methods, insufficient sample sizes, low methodological quality, and the opposite experimental subclinical control regimen, the efficacy is quite controversial.

Previous research by the research team showed that the Tuina manipulation can significantly alleviate the pain of patients with knee osteoarthritis, improve the walking ability of patients, improve joint dysfunction, and find that the work efficiency of quadriceps is significantly improved囚and the balance coordination ability of knee flexors and extensors get obviously improvement[20-21].Therefore, we designed a randomized controlled trial (RCT) to investigate whether Tuina manipulation intervention is more effective than health education intervention. Therefore, the trial will help provide reliable clinical evidence and science evidence on Tuina manipulation and massage therapy research.

\section{Methods/design}




\section{Study design}

This is a multi-center, blinded RCT with two parallel groups: the Tuina manipulation group and Health care education group. The research protocol has been approved by the Regional Ethics Review Committee of Yueyang Hospital of Integrated Traditional Chinese and Western Medicine affiliated to Shanghai University of Traditional Chinese Medicine(Project No.2019-097).It has been registered with the China Clinical Trial Registry-C-ChiCTR-2000030154. A total of 170 eligible patients with knee osteoarthritis will be randomly assigned to these groups in a 1:1 ratio. All patients will be provided with written informed consent. Outcome assessments and statistical analyses will be performed by independent investigators blinded to patient assignments. The research design is illustrated in the flow chart of Figure 1, and the research schedule is shown in Figure 2.

\section{Participants}

Eligible participants are patients diagnosed with KOA who notified of the trial if knee pain or dysfunction affects their daily activities at work or elsewhere.If patients express willingness, the clinical trial communicator will describe the trial to them and ask them if they would like to participate. If the patient wishes, the interviewee will conduct a face-to-face interview in the reception room of the hospital.Patients who meet the inclusion criteria but do not meet the exclusion criteria can join after signing the consent form.

\section{recruitment}

We made this trial public to potential participants in the following ways: 1) posting recruitment posters in the hospital's outpatient and ward corridors; 2) making roll-ups in community centers near the hospital; 3 ) the hospital's official Weibo post recruitment information; And the hospital's official WeChat platform, and 4) print ads in newspapers.

\section{Inclusion criteria}

Participants who meet all of the following criteria can be enrolled:

1) Meet the diagnostic criteria for knee osteoarthritis

2) Knee X-ray Kellgren and Lawrence grade 1 is graded: the joint space is suspected to be narrow and may have osteophytes

3) The intensity of pain measured on visual analogue scale (VAS) at the time of recruitment exceeds 3 points

4) Age between 40 and 65 years old $囚$ male or female

5) Did not receive any massage or other related treatment within the last 4 weeks of the study 
6) Those who voluntarily join the trial and sign the informed consent form

7) Ability and willingness to comply with interventions and follow-up assessments

8) Agree to not receive other related treatments (including cartilage softeners) during treatment

\section{Exclusion criteria}

Participants who meet any of the following conditions will be excluded》

1) Those with a history of trauma and surgery in the knee; patients with diseases such as tumors, tuberculosis, and osteomyelitis in the knee

2) severe liver and kidney dysfunction, severe cardiovascular disease, diabetes and mental illness affect the massage therapy

3) unable to communicate or provide self-care due to mental or psychological disorders associated with severe neurosis or dementia

4) Those with physical pain caused by other diseases

5) Partial damage in acute phase or local skin damage

6) The duration of knee-joint pain or stiffness episode is less than 0.5 hours

7) Pain attack occurs less than 3 months or more than 2 years

8) Failure to adhere to treatment at the prescribed time

9) Use massage or other related treatments within one week before entering the study

10) Participate in other clinical trials

11) Use any other treatment (drug or non-drug) in the middle

\section{Dropout criteria}

Participants who have not completed the clinical program for the following reasons should be considered as exited

1) Patient withdrawal (poor efficacy or adverse reactions)

2) Loss of follow-up

3) The researcher removes the patient (poor compliance, complications or serious adverse events) 
The test communicator will have in-depth communication with the patient who wants to withdraw from the trial because of low effectiveness or lack of time to promote retention.

\section{Randomization}

In this trial, Yueyang Hospital Science and Technology Department will generate a random sequence using a random number generator (SPSS 21.0, SPSS Inc., Chicago, IL, USA). Random numbers will be placed in opaque envelopes, numbered sequentially, and sent to therapists. Envelopes are opened in numerical order to determine the assignment of participants through the screening test. After completing the baseline questionnaire, the therapist exposes the post-physical examination and after the exclusion criteria, so that the study administrator, therapist, and the study participant are unaware of the group assignment until all baseline data has been collected.

\section{Blinding}

As an opening clinical trial, both of clinicians and patients know that they will receive which treatment and need to work with their physician or therapist before treatment. The clinical efficacy assessment will be conducted by the clinical assessor on the phone, and the assessor will conceal the treatment task. During the data collection and analysis phase, clinical researchers, evaluators, and statisticians do not share research information with each other.

\section{Interventions}

Participants will receive 8 treatments within 4 weeks. The treatment will be carried out by a senior therapist who has studied acupuncture and massage and has been licensed for more than 10 years.Participants will be asked to rest for 15 minutes before treatment.A constant room temperature of 23 to $25^{\circ} \mathrm{C}$ will be maintained to ensure that the patient is comfortable and relaxed during treatment. The intervention will be done twice a week. Each treatment will last for 20 minutes. Knee function will be assessed at baseline and 2, 4, 8, 12 weeks after baseline assessment.

\section{Tuina manipulation group}

In this part of the study, the therapist will use a two-step approach to relieve pain around the knee and improve knee dysfunction by releasing soft tissue around the knee by inducing general relaxation while addressing specific structural problems. It is determined by the clinician that it may be helpful to the patient's KOA, or by promoting air transport (activation of blood circulation according to TCM theory). The specific scheme used is as follows.

The specific implementation method of massage, that is, based on the conventional acupoint massage around the knee joint of the patient, the pain point is searched by the patient description and the palpation of the surgeon, and the pain point is treated with sputum, 20 minutes each time, 3 times a week, and the course of treatment is 4 weeks. 


\section{Step one: Check pain point around the knee joint}

The patient is supine, the examiner holds the upper part of the patient's knee joint in one hand, and the thumb of the other hand is in the inner knee, the outer knee eyes, the adductor nodules, the medial and lateral joint space, the femur and tibia, the periorbital and the axillary fossa. Sliding and pressing to find pain points of these places.

When checking the popliteal fossa, take the prone position and bend the knee, and then slide and press the center of the popliteal, the lower part of the popliteal囚the outside and in the upper of the popliteal. When inspecting the inner side of the apex patellae, the examiner needs to push the patella downwards by left hand, so that the apex patellae is lifted up, with the thumb of your right hand facing upward and press facies medialis of the apex patellae with examiner's fingertips .

\section{Step two:tuina local manipulation}

The patient is placed in the supine position. The doctor stands on the affected side, tuina manipulate on a regular acupuncture point around the patient's knee joint】and then use press and rub manipulation operate on the patient's pain point. The pain point is generally considered as reflecting the underlying condition and is often used for pain relief.Press and rub manipulation frequency reach 120-140 times / min. Then patient is placed in the prone position, doctor repeat the above operation.

\section{Health Care Education Group}

The specific implementation method of health care education, the patients with knee osteoarthritis to be included, participate in a 45-minute health care education lecture three times a week for 4 weeks. The program includes a 30-minute lecture and a 15-minute discussion. The content includes diagnosis of knee osteoarthritis, healthy diet\pain management, physical and mental health, correct lifestyle.

\section{Allowance of concurrent treatment of patients}

All KOA treatments are prohibited during the trial, including oral muscle relaxants, anesthetics, analgesics, surgery, drug injections, acupuncture and physical therapy. They may receive any treatment that is not related to knee pain. Any changes to concurrent treatment are recorded for each visit.

\section{Primary outcome measurements}

\section{KOOS}

KOOS has been widely used in research and has been shown to have the efficiency and reliability of neck pain measurements [22]. We used a knee injury and osteoarthritis outcome score (KOOS) pain test (0-100 scale, 0 for extreme knee problems, 100 for knee-free problems) as our primary at baseline and at the end of the intervention. Clinical results. The KOOS scale is an extension of WOMAC, a self-administered 42 questionnaire covering five patient-related dimensions: pain, other disease-specific symptoms, daily functional activities, motor and recreational functions, and knee-related Quality of Life. The scale is an 
effective, reliable, and responsive method for measuring results with good test reliability and structural validity for total joint replacement. We used other KOOS subscale scores as the primary outcome.

\section{Secondary outcome measurements}

\section{MPQ}

The MPQ(McGill Pain questionnaire) is a very common scale that measures and evaluates various pains [23-24]. The MPQ contains 4 types of 20 pain descriptions. Each group of words is arranged in increasing order. One to ten groups are sensory, 11 to 15 are affective, and 16 are Evaluation, 17 to 20 groups are other related classes (miscellaneous). The test subject selects a word with the same degree of pain in each group of words (no suitable one can not be selected)

\section{SF-36}

The SF-36 questionnaire assessed the association between health-related quality of life and various factors [25]. It is widely used in the field of quality of life measurement, clinical trial evaluation and health policy assessment. It consists of 36 questions, divided into Physical Functioning (PF), Role-Physical (RP), Bodily Pain (BP): General Health (GH), Vitality, social function (SF), Role Emotional, (RE) and mental health $(\mathrm{MH})$ General Health(GH)and other eight aspects. For each domain, the score ranges from 0 to 100 , with higher scores reflecting a better quality of life. The SF-36 will be evaluated using repeated longitudinal analysis.

\section{Safety evaluation}

Treatment safety will be assessed by researchers monitoring patient symptoms before and after treatment. In this trial, adverse events (AEs), the following events will be defined as adverse events: (1) severe pain; (2) syncope; (3) deterioration in walking function; (4) caused hospitalization; (5) life Changes in threats $\llbracket$ all will be carefully recorded in the case report form.For any AEs, whether or not it is related to this treatment course, treatment should be stopped immediately. The patient should be treated and the AEs will be reported to the responsible unit and the ethics committee to determine if the patient should withdraw from treatment.

\section{Follow-up}

To assess short-term efficacy, long-term efficacy, and safety of interventions, we will track participants for 2 months after the trial. Within 2 months of unsupervised follow-up, no participants will receive special treatment other than regular knee care. At Weeks 8 and 12, the results evaluators will investigate the recurrence of their neck pain by telephone participants. Patients can also inform the assessor of their clinical signs and mood changes by email, text message or WeChat at the relevant time.During the followup period after the trial, any uncomfortable health issues will be assessed and the committee will be notified for a decision. 


\section{Data collecting and monitoring}

Results The assessor will record the clinical results in the Case Report Form (CRF), the treatment effect assessment of the intervention, and the physiological function and details of the adverse events. The completed CRF is reviewed by a four-person steering committee composed of the heads of the four research centers and submitted to the data stewards who are independent of the research team, do not know the grouping, use data entry and manage an Excel database. All data administrators have data analysis qualifications and unified training. To ensure the accuracy of the data, the two data administrators independently enter the information and perform proofreading. If there is a problem with the information in the CRF, the data administrator can fill out the lookup table and then the data administrator can modify the data according to any revisions of the Steering Committee. After confirming that the database is correct, the steering committee, data stewards, and statisticians will lock the database.

\section{Health economics}

All costs associated with this test will be recorded. These costs mainly include medical costs for direct treatment of KOA, such as inpatient bed fees, outpatient registration fees, Tuina treatment fees, daily care and testing fees, and in addition, the cost of treatment for any adverse event will be recorded and included in health costs evaluation.

\section{Sample size calculation}

The rate of the expectation of the effectiveness in Tuina manipulation group is $70 \%$ and the efficacy rate for health education is $45 \%$ based on the literature. The sample size was calculated with a significance level of 0.05 and power of 0.90 , which also considering a maximum dropout tolerance of $10 \%$. At last, 170 patients are needed for the trial, with 85 for each group in the trail center.

\section{Data analysis}

Demographic and baseline data will be analyzed with standard, descriptive statistics. Between-group differences will be tested using repeated measures analysis of variance. The acceptable significance level for all analyses was $P<0.05$. We will perform the entire data analysis process by statisticians who are independent of the research team and have no knowledge of the group settings.Data analysis was performed using SPSS software (for Microsoft Windows ${ }^{\circledR}$ SPSS, Inc., SPSS 21.0 KO, Chicago, Illinois, USA).

\section{quality control}

In order to improve the reliability of the results of this study, we designed quality control procedures from the following aspects: (1) Baseline homogeneity: This study limits the age of participants to between 40 and 65, and we will assess people with similar living habits; (2) Tuina manipulation: In this study, all researchers will be trained to fully understand the design and process of the trial, the use of CRF, and the 
control of the manipulation. To avoid operational differences, two experienced doctors received specialized standardized training before the trial and performed all treatments in accordance with standard operating procedures. In addition, during the process of treatment, except for seeking pain points, unnecessary communication between the therapist and the patient is prohibited; (3) Pain point: considering the importance of pain point seeking for accurate treatment evaluation, the pain threshold at the tenderness point will be measured after each treatment to evaluate whether there is a significant difference in stimulus and pain perception before and after each treatment, which will help to rule out the bias of pain point.

\section{Discussion}

Knee pain is one of the most challenging public health problems in the world. Given its prevalence, the need to assess effective methods of knee joint disease are important. Tuina is one of the most important parts of Chinese medicine and has contributed to the health of the Chinese people for more than a thousand years. A large body of scientific evidence supports this treatment for knee pain,but its efficacy has not been determined. Therefore, it is important to provide strong evidence about the use of massage therapy for KOA.

This trial is a comparative study of the efficacy and safety of massage (intervention) and health education (control) for pain relief and functional recovery in KOA patients. For high quality RCT, proper control is critical. Both of pain intensity and motion function are subjective, we will use validated scales and questionnaires to assess clinical outcomes. KOOS will be used as the primary result, which measures knee pain and impaired daily function. The secondary outcomes are the MPQ, which measures the intensity of pain in general and the emotional state of pain effects囚and the SF-36囚 measuring healthrelated changes in quality of life.

The A-Shi point in Chinese medicine refers to the part on the surface of the body. When it is lightly pressed, it will reproduce the specific pain being treated. Its position indicates the exact location where the Qi and blood are blocked. Manipulating at the A-Shi point is to promote the free movement of Qi and improve the blood circulation in the area, eliminate the obstacles there, and achieve the purpose of relieving pain.Studies have shown that one of mechanism of tuina seems to be the activation of piezos electric channel as a physical stimulus, and then the membrane potential changes due to the flow of charged ions to generate an analgesic effect[26]. Strength training around the joint muscles and lowintensity aerobic exercise can relieve pain. Maintaining joint mobility and improving joint function can control the progression of disease in KOA. Tuina is a treatment that uses of the doctor's manipulation to achieve muscle stimulation and joint activity. The RCT design and methodological rigor of the trial will allow the collection of valuable and high-quality data to assess the efficacy of Tuina manipulation and health education for the treatment of KOA. The trial will provide a solid foundation for KOA's clinical treatment and future research on Tuina and massage therapy.

\section{Trial Status}


Participant recruitment started in March 2020 and is expected to end in February 2021.This trial was registered in Chinese Clinical Trial Registry on 24 February 2020. The registration number is ChiCTR2000030154.

\section{Abbreviations}

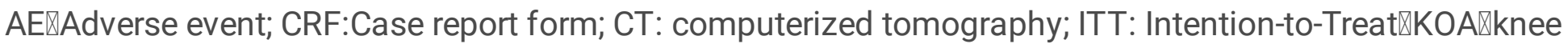
osteoarthritis $₫$ KOOS: Knee Injury and Osteoarthritis Outcome Score; MPQ: McGill pain questionnaire; MRI: Magnetic Resonance Imaging; NSAIDs: Nonsteroidal anti-inflammatory drugs; RCT: Randomized controlled trial; SF-36:36-item Short-Form Health Survey; SPSS: Statistical Package for Social Sciences; TCM: Traditional Chinese Medicine区TNF-a冈tumor necrosis factor-a; WOMAC: The Western Ontario and McMaster Universities Arthritis Index

\section{Declarations}

\section{Funding}

This study is supported financially by Shanghai further accelerate the three-year action plan for the development of TCM of Shanghai(ZY(2018-2020)-CCCX-2004-02), Major Disease Clinical Skills Upgrading Project of Shanghai Shen-kang Hospital Development Center(16CR1023A),National Major and Difficult Diseases Collaborative Pilot Project of Traditional Chinese and Western Medicine-Osteoarticular Degenerative Disease(ZY(2018-2020)-FWTX-2005),Outstanding Young Medical Talents of Shanghai Municipal Commission of Health and Family Planning(2018YQ04), Chinese Traditional Medicine Association Young Talent Project(QNRC2-A01), Chinese Medicine Research Tasks of Shanghai Health Planning Committee(2018LP040), The funders had no role in the design of the study, analysis, collection, and interpretation of the data, or the writing and decision for publication of the manuscript.All these funding apply to this study.

\section{Authors' contributions}

ZL and GG participated in the protocol and design of the trial,LK drafted the manuscript.QZ performed Tuina manipulation treating on the patients.WS recruited and screened qualified participants in the outpatient department, and MF was responsible for the study.YC is responsible for coordinating the treatment of patientsZW participated in design trials and helped prepare the manuscript. SX participated in evaluating efficacy and collecting clinical data. XZ participated in the strict revision of the manuscript.SZ participated in evaluating efficacy and collecting clinical data.CY participated in the strict proofreading of the manuscript.All authors have read and discussed the final manuscript $\$ and all approved the agreement for publication.

\section{Ethics approval and consent to participate}


This study will be conducted in accordance with the guidelines and principles of the Declaration of Helsinki.The protocol has been approved by the Ethics Review Committee of Yueyang Hospital of Integrated Traditional Chinese and Western Medicine,which is affiliated with Shanghai University of Traditional Chinese Medicine(reference 2019-097) and registered with the Chinese Clinical Trial Registry(ID: ChiCTR-2000029405).Informed consent from all study participants will be obtained before clinical trial communicators begin collecting any data. All participants will agree in writing. No one except investigators has access to the final data.

\section{Availability of data and material}

The datasets analyzed in the whole study can be obtained from the corresponding authors upon reasonable request.

\section{Consent for publication}

Not Applicable.

\section{Competing interests}

The authors declare that they have no competing interests.

\section{Author details}

${ }^{1}$ Tuina Department, Yueyang Hospital of Integrated Traditional Chinese and Western Medicine, Shanghai University of Traditional Chinese Medicine,Shanghai 200437, China. ${ }^{2}$ Institute of Tuina, Shanghai Institute of Traditional Chinese Medicine, Shanghai 200437, China. ${ }^{3}$ Shanghai University of Traditional Chinese Medicine, Shanghai 201203, China.

\section{References}

1. White AG, Birnbaum HG, Janagap C, et al. Direct an indirect costs of pain therapy for osteoarthritis in an insured population in the United States. J Occup Environ Med. 2008;50:998-1005.

2. Ong KL, Runa M, Lau E, et al. Cost-of-illness of knee osteoarthritis: potential cost savings by not undergoing arthroplasty within the frst 2 years .Clinicoecon Outcomes Res. 2019 Mar 14;11:245-255.

3. Callahan LF『Shreffler J『Siaton BC囚et al.Limited educational attainment and radiographic and symptomatic knee osteoarthritis: a cross-sectional analysis using data from the Johnston County (North Carolina) Osteoarthritis Project. Arthritis Res Ther. 2010;12(2):R46.

4. Peat G, McCarney R, Croft P. Knee pain and osteoarthritis in older adults: a review of community burden and current use of primary health care. Ann Rheum Dis. 2001;60:91-7

5. Le TK, Montejano LB, Cao Z, et al. Health care costs in US patients with and without a diagnosis of osteoarthritis. J Pain Res. 2012;5:23-30. 
6. David D McErlain,Veronica Ulici,Mark Darling,et,al.An in vivo investigation of the initiation and progression of subchondral cysts in a rodent model of secondary osteoarthritis.Arthritis Research \& Therapy (Online),2012,14(1):R26.

7. S Botha-Scheepers,I Watt,E Slagboom,et,al.Innate production of tumour necrosis factor a and interleukin 10 is associated with radiological progression of knee osteoarthritis. Ann Rheum Dis,2008,67(8):1165-1169.

8. W Zhang,M Doherty,G Peat,et al.EULAR evidence-based recommendations for the diagnosis of knee osteoarthritis. Ann Rheum Dis,2010,69(3):483-489.

9. Bozic KJ, Stacey B, Berger A, et al. Resource utilization and costs before and after total joint arthroplasty.BMC Health Serv Res.2012;12:73.

10. Cohen JR, Bradley AT, Lieberman JR. Preoperative interventions and Charges before total knee arthroplasty. J Arthroplasty. 2016;31(12):e2737:2730-2735

11. Bijlsma JW, Berenbaum F, Lafeber FP. Osteoarthritis:an update with relevance for clinical practice. Lancet.2011;377(9783): 2115 -2126.

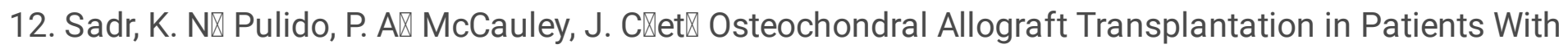
Osteochondritis Dissecans of the Knee. Am J Sports Med,2016,44(11): 2870-2875.

13. Lanas Á, Carrera-Lasfuentes $P$, Arguedas $Y$, et al. Risk of upper and lower gastrointestinal bleeding in patients taking nonsteroidal anti-inflammatory drugs, antiplatelet agents, or anticoagulants.Clin Gastroenterol Hepatol 2015;13:906-912.

14. Wallace JL. NSAID gastropathy and enteropathy: distinct pathogenesis likely necessitates distinct prevention strategies. Br J Pharmacol 2012;165:67-74.

15. Da Costa Bruno R,Nüesch Eveline,Kasteler Rahel,et al. Oral or transdermal opioids for osteoarthritis of the knee or hip. The Cochrane database of systematic reviews,2014(9).CD003115.

16. Goats GC. Massage-the scientific basis of an ancient art: Part 1: the techniques. Br J Sports Med. 1994, 28:149-52.

17. Ali A, Kahn J, Rosenberger L.Development of a manualized protocol of massage therapy for clinical trials in osteoarthritis. Trials. 2012, 13:185.

18. Ali A囚Rosenberger L『Weiss TR囚Milak C囚et al.Massage Therapy and Quality of Life in Osteoarthritis of the Knee: A Qualitative Study. Pain Med. 2017 Jun 1;18(6):1168-1175.

19. Perlman A囚Fogerite SG囚Glass O『et al.Efficacy and Safety of Massage for Osteoarthritis of the Knee: a Randomized Clinical Trial. J Gen Intern Med. 2019,34(3):379-386.

20. Zhu QG, Fang M, Gong L, et al.Gait analysis of patients with knee osteoarthritis before and after Chinese Massage treatment. Journal of Traditional Chinese Medicine, 2015,35(4): 411-416.

21. Zhu QG, Li JH, Fang M, et al.Isokinetic muscle strength evaluation of patients of knee osteoarthritis before and after Chinese Massage. Journal of Traditional Chinese Medicine, 2016ه36(3):314-320.

22. Roos EM, Toksvig-Larsen S.Knee injury and Osteoarthritis Outcome Score (KOOS)dvalidation and comparison to the WOMAC in total knee replacement. Health Qual Life Outcome 2003®1凹 
23. Vânia Tie Koga Ferreira,Elaine Caldeira de Oliveira Guirro,Almir Vieira Dibai-Filho,et al. Characterization of chronic pain in breast cancer survivors using the McGill Pain Questionnaire. Journal of Bodywork \& Movement Therapies,2015,19(4).

24. Hansen Rie Bager,Frost Charlotte Ørsted,Sonne Nan Margrethe,et al. Exploring the Patients' Perception of Background and Breakthrough Pain: A McGill Pain Questionnaire Inquiry in Patients with Bone Cancer Pain. Journal of palliative medicine,2019,22(8).

25. Lam CL, Tse EY, Gandek B, et al. The SF-36 summary scales were valid, reliable,and equivalent in a Chinese population. Clin Epidemiol. 2005;58:815-

26. Song PF, Lin ZG, Zhu QG,et al.Possible mechanism underlying analgesic effect of Tuina in rats may involve piezo mechanosensitive channels within dorsal root ganglia axon. J Tradit Chin Med 2018,38(6): 834-841.

\section{Figures}

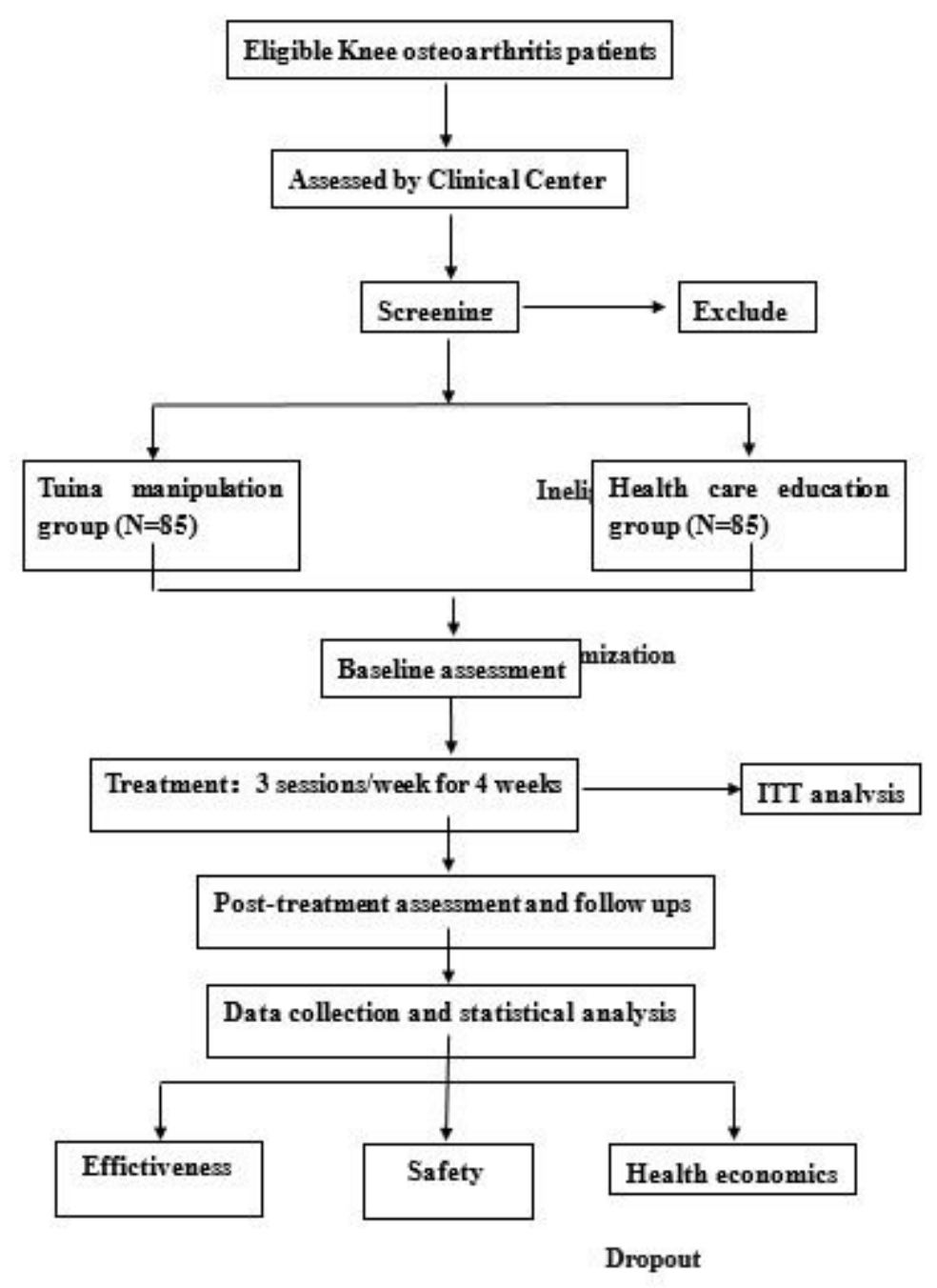

Figure 1 
Trial flow chart. This study was a single-center, randomized, health education-controlled, open-label trial.It is expected that 170 eligible KOA patients will be randomly assigned to a Tuina manipulation group or a health care education control group at a 1: 1 ratio.Patients in the Tuina manipulation group received twostep Tuina treatment including pain point examination and relaxation manipulation.Tuina manipulation treatment includes 12 treatments, each lasting about 20 minutes, 3 times a week for a total of 4 weeks.Patients in the health education group are received lectures.After collecting data,statisticians analyze the effectiveness, safety, and health economy of Tuina manipulation compared to health care education. The complete analysis set, including dropouts, will be analyzed through an intention-to-treat (ITT) population analysis.

\begin{tabular}{|c|c|c|c|c|c|c|c|c|}
\hline Tinse & w-1 & we & wi & $w_{2}$ & w] & w4 & we & w12 \\
\hline 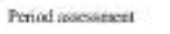 & Satening & Howite & \multicolumn{4}{|c|}{ Inbontraice } & \multicolumn{2}{|c|}{ Folluw-ip } \\
\hline \multicolumn{9}{|l|}{ Patienth dagnosic } \\
\hline Inclasustoconfrined & $\checkmark$ & & & & & & & \\
\hline Itfiented onsth & $\sqrt{ }$ & & & & & & & \\
\hline Dreabetivary & $\checkmark$ & & & & & & & \\
\hline Treanant tiskry & $\checkmark$ & & & & & & & \\
\hline Camstialy & $\checkmark$ & & & & & & & \\
\hline$x-a y$ & $\sqrt{ }$ & & & & & $\checkmark$ & $v$ & $\checkmark$ \\
\hline Fresed exutiation & $v$ & & & $\sqrt{ }$ & & $\checkmark$ & v & $v$ \\
\hline Rutibutiatat & & $\checkmark$ & & & & & & \\
\hline \multicolumn{9}{|l|}{ Iresturatiat } \\
\hline 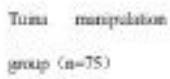 & & & \multicolumn{4}{|c|}{ 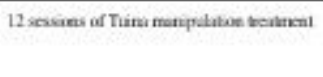 } & & \\
\hline $\begin{array}{l}\text { Heallt cate cluative } \\
\text { goup (a-75) }\end{array}$ & & & \multicolumn{4}{|c|}{ 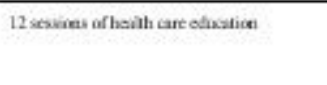 } & & \\
\hline \multicolumn{9}{|l|}{ Oreverer } \\
\hline koOs & & $\sqrt{ }$ & & $\sqrt{ }$ & & $\checkmark$ & $\sqrt{2}$ & $\sqrt{ }$ \\
\hline MFQ & & $v$ & & $\sqrt{ }$ & & $\checkmark$ & $v$ & $v$ \\
\hline
\end{tabular}

\begin{tabular}{|c|c|c|c|c|c|c|c|c|}
\hline$\$ 5.36$ & & $\sqrt{ }$ & & $\checkmark$ & & $\checkmark$ & v & $\sqrt{ }$ \\
\hline \multicolumn{9}{|c|}{ Duta wilorive iad satistical amolysis } \\
\hline Atreane event & & & $v$ & $\checkmark$ & $\checkmark$ & $\checkmark$ & v & $\checkmark$ \\
\hline 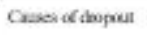 & & & $\checkmark$ & $\checkmark$ & $\checkmark$ & $v$ & $\checkmark$ & $\checkmark$ \\
\hline Sufty amolysiti & & & $\checkmark$ & $\checkmark$ & $\checkmark$ & $\checkmark$ & $\checkmark$ & $\checkmark$ \\
\hline 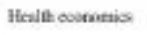 & & & & & & $\sqrt{ }$ & $\checkmark$ & $\sqrt{ }$ \\
\hline Arezòsis & $\sqrt{ }$ & $\sqrt{ }$ & $\sqrt{ }$ & $\sqrt{ }$ & $\sqrt{ }$ & $\sqrt{ }$ & $\sqrt{ }$ & $\sqrt{ }$ \\
\hline
\end{tabular}

\section{Figure 2}


Study schedule data collection showing time points for enrollment and assessment. The informed consent and examination will be conducted after recruitment.After that, we randomly matched KOA patients into two groups, one group receiving Tuina manipulation treatment and the other receiving health care education. Clinical results will be performed at two time points, including: baseline and end of session of study. During the study, adverse events were recorded on the case report form.KOOS Knee Injury and Osteoarthritis Outcome Score『MPQ McGill pain questionnaire『SF-36 36-item Short-Form Health Survey, W-1 screening before enrollment, W0 baseline assessment, W2 assessment after the sixth treatment, which is in the second week, W4 assessment after the 12th treatment, which is in the fourth week, W8 assessment 8 weeks after the first treatment, W12 assessment 12 weeks after the first treatment.

\section{Supplementary Files}

This is a list of supplementary files associated with this preprint. Click to download.

- SPIRIT.doc 